\title{
Mixing in high-dimensional expanders
}

\author{
Ori Parzanchevski (†)
}

October 25, 2013

\begin{abstract}
We prove a generalization of the Expander Mixing Lemma for arbitrary (finite) simplicial complexes. The original lemma states that concentration of the Laplace spectrum of a graph implies combinatorial expansion (which is also referred to as mixing, or quasi-randomness). Recently, an analogue of this Lemma was proved for simplicial complexes of arbitrary dimension, provided that the skeleton of the complex is complete. More precisely, it was shown that a concentrated spectrum of the simplicial Hodge Laplacian implies a similar type of expansion as in graphs. In this paper we remove the assumption of a complete skeleton, showing that concentration of the Laplace spectra in all dimensions implies combinatorial expansion in any complex. As applications we show that spectral concentration implies Gromov's geometric overlap property, and can be used to bound the chromatic number of a complex
\end{abstract}

\section{Introduction}

The spectral gap of a finite graph $G=(V, E)$ is the smallest nontrivial eigenvalue of its Laplacian operator. The discrete Cheeger inequalities [Dod84, Tan84, AM85, Alo86] relate the spectral gap to expansion in the graph: If the spectral gap is large, then for any partition $V=A \cup B$ there is a large number of edges connecting a vertex in $A$ with a vertex in $B$. Nevertheless, a large spectral gap does not suffice to control the number of edges between any two sets of vertices. For example, there exist "bipartite expanders" (see e.g. [LPS88, MSS13]): graphs with a large spectral gap, which are bipartite, so that there are $A, B \subseteq V$ of size $|A|=|B|=\frac{|V|}{4}$ with no edges between them. The Expander Mixing Lemma [FP87, AC88, BMS93] remedies this inconvenience, using not only the spectral gap but also the maximal eigenvalue of the Laplacian:

Theorem (Expander Mixing Lemma, [FP87, AC88, BMS93]). Let $G=(V, E)$ be a graph on $n$ vertices. If the nontrivial spectrum of the Laplacian is contained within $[k(1-\varepsilon), k(1+\varepsilon)]$, then for any two sets of vertices $A, B$ one has

$$
|| E(A, B)\left|-\frac{k}{n}\right| A|| B|| \leq \varepsilon k \sqrt{|A||B|},
$$

where $E(A, B)$ are the edges with one endpoint in $A$ and the other in $B$.

If $k$ is the average degree of a vertex in $G$, then $\frac{k}{n}|A||B|$ is about the expected size of $|E(A, B)|$ (the exact value is $\frac{k}{n-1}|A||B|$ ). Thus, the Lemma means that a concentrated spectrum indicates

\footnotetext{
${ }^{(\dagger)}$ Supported by The Fund for Math at the Institute for Advanced Study.
} 
a quasi-random behavior. In light of the Expander Mixing Lemma, we call a graph whose nontrivial Laplace spectrum is contained in $[k(1-\varepsilon), k(1+\varepsilon)]$ a $(k, \varepsilon)$-expander ${ }^{(\dagger)}$.

In [PRT13] a generalization of the Expander Mixing Lemma was proved for simplicial complexes of arbitrary dimension, assuming that they have a complete skeleton ${ }^{(\ddagger)}$. The Laplace operator which is studied there and in the current paper originates in Eckmann's work [Eck44]. It is a natural analogue of the Hodge Laplace operator in Riemmanian geometry, and it was studied in several prominent works [Gar73, Żuk96, Fri98, KRS00, ABM05, DKM09], sometimes under the name combinatorial Laplacian. More precisely, a complex of dimension $d$ has $d$ Laplace operators (defined here in $\S 2$ ), with the $j$-th one acting on the cells of dimension $j(0 \leq j<d)$. We say that $X$ is a $(j, k, \varepsilon)$-expander if $\varepsilon<1$, and the nontrivial spectrum of the $j$-th Laplacian is contained in $[k(1-\varepsilon), k(1+\varepsilon)]$ (see $\S 2.1$ for the precise definition).

Theorem ([PRT13]). Let $X$ be a d-complex on $n$ vertices with a complete skeleton, which is a $(d-1, k, \varepsilon)$-expander. For any disjoint $A_{0}, \ldots, A_{d} \subseteq V$,

$$
|| F\left(A_{0}, \ldots, A_{d}\right)\left|-\frac{k}{n}\right| A_{0}|\cdot \ldots \cdot| A_{d}|| \leq \varepsilon k\left(\left|A_{0}\right| \cdot \ldots \cdot\left|A_{d}\right|\right)^{\frac{d}{d+1}}
$$

where $F\left(A_{0}, \ldots, A_{d}\right)$ is the set of $d$-cells with one vertex in each $A_{i}$.

In this paper we prove a mixing lemma for arbitrary (finite) complexes. Our main result is the following (this is a special case of Proposition 3.1):

Theorem 1.1. If a d-dimensional complex $X$ is a $\left(j, k_{j}, \varepsilon_{j}\right)$-expander for every $0 \leq j \leq d-1$, and $A_{0}, \ldots, A_{d}$ are disjoint sets of vertices in $X$ then

$$
|| F\left(A_{0}, \ldots, A_{d}\right)\left|-\frac{k_{0} \ldots k_{d-1}}{n^{d}}\right| A_{0}|\cdot \ldots \cdot| A_{d}|| \leq c_{d} k_{0} \ldots k_{d-1}\left(\varepsilon_{0}+\ldots+\varepsilon_{d-1}\right) \max \left|A_{i}\right|,
$$

where $c_{d}$ depends only on $d$.

In order to understand $F\left(A_{0}, \ldots, A_{d}\right)$ in the case of general complexes, we study a wider counting problem:

Definition 1.2. Given disjoint sets $A_{0}, \ldots, A_{\ell} \subseteq V$, and $j \leq \ell$, a $j$-gallery in $A_{0}, \ldots, A_{\ell}$ is a sequence of $j$-cells $\sigma_{0}, \ldots, \sigma_{\ell-j} \in X^{j}$, such that $\sigma_{i}$ is in $F\left(A_{i}, \ldots, A_{i+j}\right)$, and $\sigma_{i}$ and $\sigma_{i+1}$ intersect in a $(j-1)$-cell (which must lie in $\left.F\left(A_{i+1}, \ldots, A_{i+j}\right)\right)$. We denote the set of $j$-galleries in $A_{0}, \ldots, A_{\ell}$ by $F^{j}\left(A_{0}, \ldots, A_{\ell}\right)$.

\section{Example.}

(1) An $\ell$-gallery in $A_{0}, \ldots, A_{\ell}$ is just a single $\ell$-cell, so that $F^{\ell}\left(A_{0}, \ldots, A_{\ell}\right)=F\left(A_{0}, \ldots, A_{\ell}\right)$.

(2) A 0 -gallery is any sequence of vertices, so that $F^{0}\left(A_{0}, \ldots, A_{\ell}\right)=A_{0} \times \ldots \times A_{\ell}$.

(3) $F^{2}(A, B, C, D, E)$ is the number of triplets of triangles $t_{1} \in F(A, B, C), t_{2} \in F(B, C, D)$, $t_{3} \in F(C, D, E)$ such that the boundaries of $t_{1}$ and $t_{2}$ share a common edge (necessarily in $F(B, C)$ ), and likewise for $t_{2}$ and $t_{3}$.

${ }^{(\dagger)}$ In [Tao11] this is referred to as a "two-sided $(k, \varepsilon)$-expander", as the spectrum is bounded on both sides.

(‡) A $d$-dimensional complex is said to have a complete skeleton if every cell of dimension smaller than $d$ is in the complex. For example, a triangle complex with a complete underlying graph. Such complexes are sometimes called hypergraphs. 
The heart of our analysis is the following lemma, which estimates the size of $F^{j+1}\left(A_{0}, \ldots, A_{\ell}\right)$ in terms of that of $F^{j}\left(A_{0}, \ldots, A_{\ell}\right)$. Repeatedly applying this lemma allows us to estimate $\left|F\left(A_{0}, \ldots, A_{d}\right)\right|=\left|F^{d}\left(A_{0}, \ldots, A_{d}\right)\right|$ in terms of $\left|F^{0}\left(A_{0}, \ldots, A_{d}\right)\right|=\left|A_{0}\right| \cdot \ldots \cdot\left|A_{d}\right|$.

Lemma 1.3 (Descent Lemma). Let $A_{0}, \ldots, A_{\ell}$ be disjoint sets of vertices in $X$. $\left.^{\dagger}\right)$ If $X$ is an $\left(i, k_{i}, \varepsilon_{i}\right)$-expander for $i=j-1, i=j$, then

$$
\begin{array}{r}
|| F^{j+1}\left(A_{0}, \ldots, A_{\ell}\right)\left|-\left(\frac{k_{j}}{k_{j-1}}\right)^{\ell-j}\right| F^{j}\left(A_{0}, \ldots, A_{\ell}\right)|| \\
\leq(\ell-j) k_{j}^{\ell-j}\left(\varepsilon_{j}+\varepsilon_{j-1}\right) \sqrt{\left|F\left(A_{0}, \ldots, A_{j}\right)\right|\left|F\left(A_{\ell-j}, \ldots, A_{\ell}\right)\right|} .
\end{array}
$$

The proofs of this lemma and of the mixing lemma it implies (Theorem 1.1) appear in $\S 3$, after giving the required definitions in $\S 2$. In $\S 4$ we demonstrate applications of the mixing lemma, showing that spectral expanders form geometric expanders (in the sense of Gromov, see [Gro10, MW11]), and have large chromatic numbers. We also present the idea of ideal expanders in this section, and list some open problems in $§ 5$.

Acknowledgement. I would like to thank Konstantin Golubev, Alex Lubotzky, Ron Rosenthal and Doron Puder for many valuable discussions.

\section{Simplicial Hodge theory}

We describe here briefly the notions we shall need from the so-called simplicial Hodge theory, originating in [Eck44]. For a more detailed summary we refer the reader to [PRT13, §2].

Let $X$ be a $d$-dimensional simplicial complex on $n$ vertices $V$. For $-1 \leq j \leq d$ we denote by $X^{j}$ the set of $j$-cells in $X$ (cells of size $j+1$ ), and by $X_{ \pm}^{j}$ the set of oriented $j$-cells, i.e. ordered cells up to an even permutation. A $j$-form on $X$ is an antisymmetric function on oriented $j$-cells:

$$
\Omega^{j}=\Omega^{j}(X)=\left\{f: X_{ \pm}^{j} \rightarrow \mathbb{R} \mid f(\bar{\sigma})=-f(\sigma) \forall \sigma \in X_{ \pm}^{j}\right\}
$$

where $\bar{\sigma}$ is $\sigma$ endowed with the opposite orientation. In dimensions 0 and -1 there is only one

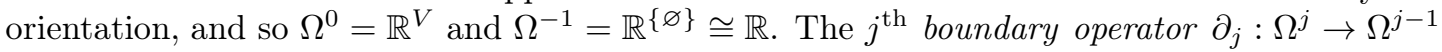
is defined by $\left(\partial_{j} f\right)(\sigma)=\sum_{v \cup \sigma \in X^{j}} f(v \sigma)$. The sequence $\Omega^{-1} \stackrel{\partial_{0}}{\longleftarrow} \Omega^{0} \stackrel{\partial_{1}}{\longleftarrow} \ldots$ is a chain complex, i.e. $B_{j} \stackrel{\text { def }}{=} \operatorname{im} \partial_{j+1} \subseteq$ ker $\partial_{j} \stackrel{\text { def }}{=} Z_{j}$, and $H^{j}=Z_{j} / B_{j}$ is the $j^{\text {th }}$ (real, reduced) homology group of $X$. We endow each $\Omega^{j}$ with the inner product $\langle f, g\rangle=\sum_{\sigma \in X^{j}} f(\sigma) g(\sigma)$, which gives rise to a dual coboundary operator $\delta_{j}=\partial_{j}^{*}: \Omega^{j-1} \rightarrow \Omega^{j}$. The real cohomology of $X$ is $H^{j}=Z^{j} / B^{j}$, where $B^{j} \stackrel{\text { def }}{=} \operatorname{im} \delta_{j} \subseteq \operatorname{ker} \delta_{j+1} \stackrel{\text { def }}{=} Z^{j}$, and by the fundamental theorem of linear algebra one has $B_{j}^{\perp}=Z^{j}$ and $Z_{j}^{\perp}=B^{j}$.

Simplicial Hodge theory, originating in [Eck44], studies the upper, lower and full Laplacians: $\Delta_{j}^{+}=\partial_{j+1} \delta_{j+1}, \Delta_{j}^{-}=\delta_{j} \partial_{j}$, and $\Delta_{j}=\Delta_{j}^{+}+\Delta_{j}^{-}$, respectively. All of the Laplacians are selfadjoint and decompose with respect to the orthogonal decompositions $\Omega^{j}=B^{j} \oplus Z_{j}=B_{j} \oplus Z^{j}$, and the following properties are simple exercises:

$$
\begin{gathered}
Z^{j}=\operatorname{ker} \Delta_{j}^{+} \quad B_{j}=\operatorname{im} \Delta_{j}^{+} \quad Z_{j}=\operatorname{ker} \Delta_{j}^{-} \quad B^{j}=\operatorname{im} \Delta_{j}^{-} \\
Z^{j} \cap Z_{j}=\left(B_{j} \oplus B^{j}\right)^{\perp}=\operatorname{ker} \Delta_{j} \cong H_{j} \cong H^{j} \quad \text { (Discrete Hodge Theorem). }
\end{gathered}
$$

\footnotetext{
${ }^{(\dagger)}$ In fact, it suffices that each $j+1$ tuple $A_{i}, A_{i+1}, \ldots, A_{i+j+1}$ consist of disjoint set.
} 
The dimension of ker $\Delta_{j} \cong H_{j} \cong H^{j}$ is the $j^{\text {th }}$ (reduced) Betti number of $X$, denoted by $\beta_{j}$.

The combinatorial meaning of the Laplacians is better understood via the following adjacency relations on oriented cells:

(1) For two oriented $j$-cells $\sigma, \sigma^{\prime}$, we denote $\sigma \pitchfork \sigma^{\prime}$ if $\sigma$ and $\sigma^{\prime}$ intersect in a common $(j-1)$ cell and induce the same orientation on it; for edges this means that they have a common origin or a common endpoint, and for vertices $v \pitchfork v^{\prime}$ holds whenever $v \neq v^{\prime}$.

(2) We denote $\sigma \sim \sigma^{\prime}$ if: $\sigma \pitchfork \sigma^{\prime}$, and in addition the $(j+1)$-cell $\sigma \cup \sigma^{\prime}$ is in $X$. For vertices this is the common relation of neighbors in a graph ${ }^{(\dagger)}$.

Using these relations, the Laplacians can be expressed as follows (here the degree of a $j$-cell is the number of $(j+1)$-cells in which it is contained):

$$
\begin{aligned}
& \left(\Delta_{j}^{+} \varphi\right)(\sigma)=\operatorname{deg}(\sigma) \varphi(\sigma)-\sum_{\sigma^{\prime} \sim \sigma} \varphi\left(\sigma^{\prime}\right) \\
& \left(\Delta_{j}^{-} \varphi\right)(\sigma)=(j+1) \varphi(\sigma)+\sum_{\sigma^{\prime} \pitchfork \sigma} \varphi\left(\sigma^{\prime}\right) \\
& \left(\Delta_{j} \varphi\right)(\sigma)=(\operatorname{deg} \sigma+j+1) \varphi(\sigma)+\sum_{\substack{\sigma^{\prime} \pitchfork \sigma \\
\sigma^{\prime} \nsim \sigma}} \varphi\left(\sigma^{\prime}\right)
\end{aligned}
$$

We shall also define adjacency operators on $\Omega^{j}$ which correspond to the $\sim$ and $\pitchfork$ relations:

$$
\left(\mathcal{A}_{j}^{\sim} \varphi\right)(\sigma)=\sum_{\sigma^{\prime} \sim \sigma} \varphi\left(\sigma^{\prime}\right), \quad\left(\mathcal{A}_{j}^{\pitchfork} \varphi\right)(\sigma)=\sum_{\sigma^{\prime} \pitchfork \sigma} \varphi\left(\sigma^{\prime}\right),
$$

so that $\Delta_{j}^{-}=(j+1) \cdot I+\mathcal{A}_{j}^{\text {巾 }}$ and $\Delta_{j}^{+}=D_{j}-\mathcal{A}_{j}^{\sim}$, where $D_{j}$ is the degree operator $\left(D_{j} f\right)(\sigma)=$ $\operatorname{deg}(\sigma) f(\sigma)$.

\section{$2.1 \quad$ Spectrum}

The spectra we are primarily interested in are those of $\Delta_{j}^{+}$for $0 \leq j \leq d-1$. Since $\left(\Omega^{j}, \delta_{j}\right)$ is a co-chain complex, $B^{j}=\operatorname{im} \delta_{j}$ must be contained in the kernel of $\Delta_{j}^{+}=\partial_{j+1} \delta_{j+1}$, and the zero eigenvalues which correspond to forms in $B^{j}$ are considered to be the trivial spectrum of $\Delta_{j}^{+}$. As $\left(B^{j}\right)^{\perp}=Z_{j}$, we call Spec $\left.\Delta_{j}^{+}\right|_{Z_{j}}$ the nontrivial spectrum of $\Delta_{j}^{+}$. Note that zero is a nontrivial eigenvalue of $\Delta_{j}^{+}$precisely when $Z_{j} \cap Z^{j} \neq 0$, i.e. $\beta_{j} \neq 0$. For example, the constant functions on $V$ form the trivial eigenfunctions of $\Delta_{0}^{+}$. The nontrivial spectrum of $\Delta_{j}^{+}$corresponds to $Z_{0}$, which are the functions whose sum on all vertices vanish, and zero is a nontrivial eigenvalue of $\Delta_{0}^{+}$iff the complex is disconnected.

As hinted in the introduction, we say that $X$ is a $(j, k, \varepsilon)$-expander if $\varepsilon<1$ and $\left.\operatorname{Spec} \Delta_{j}^{+}\right|_{Z_{j}} \subseteq$ $[k(1-\varepsilon), k(1+\varepsilon)]$. Given $\bar{k}=\left(k_{0}, \ldots, k_{d-1}\right)$ and $\bar{\varepsilon}=\left(\varepsilon_{0}, \ldots, \varepsilon_{d-1}\right)$, we say that $X$ is a $(\bar{k}, \bar{\varepsilon})$ expander if it is a $\left(j, k_{j}, \varepsilon_{j}\right)$-expander for all $j$. The restriction $\varepsilon_{j}<1$ ensures that $X$ has trivial $j$-th homology, i.e. $\beta_{j}=0$. While some of our results hold for general $\varepsilon$ (e.g. Lemma 1.3), or for any global bound on it (e.g. Theorem 1.1), we shall need the stronger assumption for later applications.

Finally, we remark that it is sometimes useful to consider the Laplacian $\Delta_{-1}^{+}$as well. This operator acts on $\Omega^{-1} \cong \mathbb{R}$ as multiplication by $n=|V|$, so that every complex is automatically a $(-1, n, 0)$-expander.

\footnotetext{
${ }^{(\dagger)}$ This adjacency relation can be used to define a stochastic process on $j$-cells whose properties relate to the homology of the complex - see [PR12].
} 


\section{The main theorems}

In this section we assume that $X$ is a $d$-complex on $n$ vertices, which is a $\left(j, k_{j}, \varepsilon_{j}\right)$-expander for $0 \leq j<d$, and prove the Descent Lemma (Lemma 1.3) and the mixing lemmas it implies.

Proof of the Descent Lemma. To any disjoint sets of vertices $A_{0}, \ldots, A_{j}$, we associate the characteristic $j$-form $\delta_{A_{0} \ldots A_{j}} \in \Omega^{j}$, which takes \pm 1 on $j$-cells in $F\left(A_{0}, \ldots, A_{j}\right)$ (according to their orientation), and vanishes elsewhere:

$$
\delta_{A_{0} \ldots A_{j}}(\sigma)= \begin{cases}\operatorname{sgn}(\pi) & \exists \pi \in \operatorname{Sym}_{\{0 \ldots j\}} \text { with } \sigma_{i} \in A_{\pi(i)} \text { for } 0 \leq i \leq j \\ 0 & \text { otherwise. }\end{cases}
$$

Multiplication by $\delta_{A_{0} \ldots A_{j}}$ forms a projection operator on $\Omega^{j}$, which we denote by $\mathbb{P}_{A_{0} \ldots A_{j}}$ :

$$
\mathbb{P}_{A_{0} \ldots A_{j}}(\varphi)=\delta_{A_{0} \ldots A_{j}} \cdot \varphi=\sigma \mapsto \delta_{A_{0} \ldots A_{j}}(\sigma) \varphi(\sigma) .
$$

We start our analysis by observing for disjoint sets $A_{0}, \ldots, A_{j+1}$ the form $(-1)^{j} \mathbb{P}_{A_{0} \ldots A_{j}} \mathcal{A}_{j}^{\sim} \delta_{A_{1} \ldots A_{j+1}}$ vanishes outside $F\left(A_{0}, \ldots, A_{j}\right)$, and to each $j$-cell therein it assigns the number of its $\sim$-neighbors in $F\left(A_{1}, \ldots, A_{j+1}\right)$. As these neighbors are in correspondence with $(j+1)$-cells in $F\left(A_{0}, \ldots, A_{j+1}\right)$, we obtain that $\left|\left\langle\delta_{A_{0} \ldots A_{j}}, \mathbb{P}_{A_{0} \ldots A_{j}} \mathcal{A}_{j}^{\sim} \delta_{A_{1} \ldots A_{j+1}}\right\rangle\right|=\left|F\left(A_{0}, \ldots, A_{j+1}\right)\right|$.

Next, let $\varphi$ be a $j$-form which is supported on $F\left(A_{1}, \ldots, A_{j+1}\right)$, and which assigns to each $j$-cell $\sigma$ the number of $(j+1)$-galleries in $A_{1}, \ldots, A_{\ell}$ whose first cell contains $\sigma$. By the same consideration as above, $(-1)^{j} \mathbb{P}_{A_{0} \ldots A_{j}} \mathcal{A}_{j}^{\sim} \varphi$ assigns to every $j$-cell $\tau$ in $F\left(A_{0}, \ldots, A_{j}\right)$ the number of $(j+1)$-galleries in $A_{0}, \ldots, A_{\ell}$ whose first $(j+1)$ cell contains $\tau$. Therefore, $\left|\left\langle\delta_{A_{0} \ldots A_{j}}, \mathbb{P}_{A_{0} \ldots A_{j}} \mathcal{A}_{j}^{\sim} \varphi\right\rangle\right|=\left|F^{j+1}\left(A_{0}, \ldots, A_{\ell}\right)\right|$, and we conclude by induction that

$$
\left|F^{j+1}\left(A_{0}, \ldots, A_{\ell}\right)\right|=\left|\left\langle\delta_{A_{0} \ldots A_{j}},\left(\prod_{i=0}^{\ell-j-1} \mathbb{P}_{A_{i} \ldots A_{i+j}} \mathcal{A}_{j}^{\sim}\right) \delta_{A_{\ell-j} \ldots A_{\ell}}\right\rangle\right| .
$$

Since the $A_{i}$ are disjoint, $\delta_{A_{i} \ldots A_{i+j}}$ and $\delta_{A_{i+1} \ldots A_{i+j+1}}$ are supported on different cells, so that $\mathbb{P}_{A_{i} \ldots A_{i+j}} T \delta_{A_{i+1} \ldots A_{i+j+1}}=0$ for any diagonal operator $T$. Thus, all the $\mathcal{A}_{j}^{\sim}$ in (3.1) can be replaced by $\mathcal{A}_{j}^{\sim}+T$, and taking $T=k_{j} I-D_{j}$ we obtain

$$
\left|F^{j+1}\left(A_{0}, \ldots, A_{\ell}\right)\right|=\left|\left\langle\delta_{A_{0} \ldots A_{j}},\left(\prod_{i=0}^{\ell-j-1} \mathbb{P}_{A_{i} \ldots A_{i+j}}\left(k_{j} I-\Delta_{j}^{+}\right)\right) \delta_{A_{\ell-j} \ldots A_{\ell}}\right\rangle\right| .
$$

Our next step is to approximate this quantity using the lower $j$-th Laplacian. Denoting $E=$ $k_{j} I-\Delta_{j}^{+}-\frac{k_{j}}{k_{j-1}} \Delta_{j}^{-}$, the orthogonal decomposition $\Omega^{j}=Z_{j} \oplus B^{j}$ gives

$$
E=k_{j}\left(\mathbb{P}_{Z_{j}}+\mathbb{P}_{B^{j}}\right)-\Delta_{j}^{+}-\frac{k_{j}}{k_{j-1}} \Delta_{j}^{-}=k_{j} \mathbb{P}_{Z_{j}}-\Delta_{j}^{+}+\frac{k_{j}}{k_{j-1}}\left(k_{j-1} \mathbb{P}_{B^{j}}-\Delta_{j}^{-}\right) .
$$

We first observe that $\left\|k_{j} \mathbb{P}_{Z_{j}}-\Delta_{j}^{+}\right\| \leq k_{j} \varepsilon_{j}$ follows from $\left.\operatorname{Spec} \Delta_{j}^{+}\right|_{Z_{j}} \subseteq\left[k_{j}\left(1-\varepsilon_{j}\right), k_{j}\left(1+\varepsilon_{j}\right)\right]$ and $\left.\Delta_{j}^{+}\right|_{B^{j}} \equiv 0$. For the lower Laplacian, we have

$$
\begin{aligned}
& \left.\operatorname{Spec} \Delta_{j}^{-}\right|_{B^{j}}=\left.\operatorname{Spec} \Delta_{j}^{-}\right|_{Z_{j}^{\perp}}=\operatorname{Spec} \Delta_{j}^{-} \backslash\{0\} \stackrel{(*)}{=} \operatorname{Spec} \Delta_{j-1}^{+} \backslash\{0\}=\left.\operatorname{Spec} \Delta_{j-1}^{+}\right|_{\left(Z^{j-1}\right)^{\perp}} \\
& =\left.\left.\operatorname{Spec} \Delta_{j-1}^{+}\right|_{B_{j-1}} \subseteq \operatorname{Spec} \Delta_{j-1}^{+}\right|_{Z_{j-1}} \subseteq\left[k_{j-1}\left(1-\varepsilon_{j-1}\right), k_{j-1}\left(1+\varepsilon_{j-1}\right)\right],
\end{aligned}
$$


where $(*)$ follows from the fact that $\Delta_{j}^{-}=\partial_{j}^{*} \partial_{j}$ and $\Delta_{j-1}^{+}=\partial_{j} \partial_{j}^{*}$. As $\Delta_{j}^{-}$vanishes on $Z_{j}$, we have in total $\left\|k_{j-1} \mathbb{P}_{B^{j}}-\Delta_{j}^{-}\right\| \leq k_{j-1} \varepsilon_{j-1}$, so that

$$
\|E\| \leq\left\|k_{j} \mathbb{P}_{Z_{j}}-\Delta_{j}^{+}\right\|+\frac{k_{j}}{k_{j-1}}\left\|k_{j-1} \mathbb{P}_{B^{j}}-\Delta_{j}^{-}\right\| \leq k_{j}\left(\varepsilon_{j-1}+\varepsilon_{j}\right) .
$$

We proceed to expand (3.2), using $\frac{k_{j}}{k_{j-1}} \Delta_{j}^{-}+E=k_{j} I-\Delta_{j}^{+}$, and on occasions translating $\Delta_{j}^{-}$ by some diagonal (in fact, scalar) operators:

$$
\begin{aligned}
& \left|F^{j+1}\left(A_{0}, \ldots, A_{\ell}\right)\right|=\left|\left\langle\delta_{A_{0} \ldots A_{j}},\left(\prod_{i=0}^{\ell-j-1} \mathbb{P}_{A_{i} \ldots A_{i+j}}\left(\frac{k_{j}}{k_{j-1}} \Delta_{j}^{-}+E\right)\right) \delta_{A_{\ell-j} \ldots A_{\ell}}\right\rangle\right| \\
& =\mid\left(\frac{k_{j}}{k_{j-1}}\right)^{\ell-j}\left\langle\delta_{A_{0} \ldots A_{j}},\left(\prod_{i=0}^{\ell-j-1} \mathbb{P}_{A_{i} \ldots A_{i+j}} \Delta_{j}^{-}\right) \delta_{A_{\ell-j} \ldots A_{\ell}}\right\rangle \\
& +\sum_{m=1}^{\ell-j}\left(\frac{k_{j}}{k_{j-1}}\right)^{\ell-j-m}\left\langle\delta_{A_{0} \ldots A_{j}}, \begin{array}{l}
\left(\prod_{i=0}^{\ell-j-m-1} \mathbb{P}_{A_{i} \ldots A_{i+j}} \Delta_{j}^{-}\right) \mathbb{P}_{A_{\ell-j-m} \ldots A_{\ell-m}} E \cdot \\
\cdot\left(\prod_{i=\ell-j-m+1}^{\ell-j-1} \mathbb{P}_{A_{i} \ldots A_{i+j}}\left(\frac{k_{j}}{k_{j-1}} \Delta_{j}^{-}+E\right)\right) \delta_{A_{\ell-j} \ldots A_{\ell}}
\end{array}\right\rangle \\
& =\mid\left(\frac{k_{j}}{k_{j-1}}\right)^{\ell-j}\left\langle\delta_{A_{0} \ldots A_{j}},\left(\prod_{i=0}^{\ell-j-1} \mathbb{P}_{A_{i} \ldots A_{i+j}} \mathcal{A}_{j}^{\dagger}\right) \delta_{A_{\ell-j} \ldots A_{\ell}}\right\rangle \\
& +\sum_{m=1}^{\ell-j}\left(\frac{k_{j}}{k_{j-1}}\right)^{\ell-j-m}\left\langle\delta_{A_{0} \ldots A_{j}}, \frac{\left(\prod_{i=0}^{\ell-j-m-1} \mathbb{P}_{A_{i} \ldots A_{i+j}}\left(\Delta_{j}^{-}-k_{j-1} I\right)\right) \mathbb{P}_{A_{\ell-j-m} \ldots A_{\ell-m} E} E \cdot \mid}{\cdot\left(\prod_{i=\ell-j-m+1}^{\ell-j-1} \mathbb{P}_{A_{i} \ldots A_{i+j}}\left(k_{j} I-\Delta_{j}^{+}\right)\right) \delta_{A_{\ell-j} \ldots A_{\ell}}}\right\rangle .
\end{aligned}
$$

We first study the summand in line (3.4). Note that the form $(-1)^{j} \mathbb{P}_{A_{0} \ldots A_{j}} \mathcal{A}_{j}^{\dagger} \delta_{A_{1} \ldots A_{j+1}}$ assigns to every $j$-cell in $F\left(A_{0}, \ldots, A_{j}\right)$ the number of $j$-cells in $F\left(A_{1}, \ldots, A_{j+1}\right)$ with which it intersects, so that $\left|\left\langle\delta_{A_{0} \ldots A_{j}}, \mathbb{P}_{A_{0} \ldots A_{j}} \mathcal{A}_{j}^{\pitchfork} \delta_{A_{1} \ldots A_{j+1}}\right\rangle\right|=\left|F^{j}\left(A_{0}, \ldots, A_{j+1}\right)\right|$ (recall that for $A_{j}^{\sim}$ in place of $A_{j}^{\pitchfork}$ we obtained $\left.\left|F^{j+1}\left(A_{0}, \ldots, A_{j+1}\right)\right|\right)$. By the same arguments as before one sees that

$$
\left|F^{j}\left(A_{0}, \ldots, A_{\ell}\right)\right|=\left|\left\langle\delta_{A_{0} \ldots A_{j}},\left(\prod_{i=0}^{\ell-j-1} \mathbb{P}_{A_{i} \ldots A_{i+j}} \mathcal{A}_{j}^{\dagger}\right) \delta_{A_{\ell-j} \ldots A_{\ell}}\right\rangle\right|,
$$

so that line (3.4) is precisely $\left(\frac{k_{j}}{k_{j-1}}\right)^{\ell-j}\left|F^{j}\left(A_{0}, \ldots, A_{\ell}\right)\right|$, our estimate for $\left|F^{j+1}\left(A_{0}, \ldots, A_{\ell}\right)\right|$. Denoting by $\mathcal{E}$ the error term (the line below (3.4)), we bound it using (3.3) together with $\left\|\Delta_{j}^{-}-k_{j-1} I\right\| \leq k_{j-1}$ and $\left\|k_{j} I-\Delta_{j}^{+}\right\| \leq k_{j}$ (both follow from the discussion preceding (3.3)):

$$
\begin{aligned}
\mathcal{E} & \leq \sum_{m=1}^{\ell-j}\left(\frac{k_{j}}{k_{j-1}}\right)^{\ell-j-m}\left\|\delta_{A_{0} \ldots A_{j}}\right\| k_{j-1}^{\ell-j-m} k_{j}\left(\varepsilon_{j-1}+\varepsilon_{j}\right) k_{j}^{m-1}\left\|\delta_{A_{\ell-j} \ldots A_{\ell}}\right\| \\
& =(\ell-j) k_{j}^{\ell-j}\left(\varepsilon_{j-1}+\varepsilon_{j}\right) \sqrt{\left|F\left(A_{0}, \ldots, A_{j}\right)\right|\left|F\left(A_{\ell-j}, \ldots, A_{\ell}\right)\right|},
\end{aligned}
$$

which concludes the proof. 
We remark that a slightly better bound is possible here: As Spec $\Delta_{j}^{+} \subseteq\left[0, k_{j}\left(1+\varepsilon_{j}\right)\right]$, we can replace $k_{j} I-\Delta_{j}^{+}$in the line below (3.4) by $\frac{k_{j}\left(1+\varepsilon_{j}\right)}{2} I-\Delta_{j}^{+}$, which is bounded by $\frac{k_{j}\left(1+\varepsilon_{j}\right)}{2}$, and likewise for $\Delta_{j}^{-}$(whose spectrum lies within $\left[0, k_{j-1}\left(1+\varepsilon_{j-1}\right)\right]$ ). For example, putting $\varepsilon=\max \varepsilon_{i}$ this gives

$$
\mathcal{E} \leq(\ell-j) k_{j}^{\ell-j} 2 \varepsilon\left(\frac{1+\varepsilon}{2}\right)^{\ell-j-1} \sqrt{\left|F\left(A_{0}, \ldots, A_{j}\right)\right|\left|F\left(A_{\ell-j}, \ldots, A_{\ell}\right)\right|}
$$

which might be useful when all $\varepsilon_{i}$ are small.

Using the Descent Lemma repeatedly gives:

Proposition 3.1. For any $j<\ell$, there exists $c_{j, \ell}$ such that any disjoint sets of vertices $A_{0}, \ldots, A_{\ell}$ in a $(\bar{k}, \bar{\varepsilon})$-expander satisfy

$$
|| F^{j+1}\left(A_{0}, \ldots, A_{\ell}\right)\left|-\frac{k_{0} k_{1} \ldots k_{j-1} k_{j}^{\ell-j}}{n^{\ell}} \prod_{i=0}^{\ell}\right| A_{i}|| \leq c_{j, \ell} k_{0} k_{1} \ldots k_{j-1} k_{j}^{\ell-j}\left(\varepsilon_{0}+\ldots+\varepsilon_{j}\right) \max \left|A_{i}\right| .
$$

In particular, for $j=d-1, \ell=d$ we obtain Theorem 1.1:

Theorem (Theorem 1.1). Any disjoint sets of vertices $A_{0}, \ldots, A_{d}$ in a $(\bar{k}, \bar{\varepsilon})$-expander of dimension d satisfy

$$
|| F\left(A_{0}, \ldots, A_{d}\right)\left|-\frac{k_{0} \ldots k_{d-1}}{n^{d}}\right| A_{0}|\cdot \ldots \cdot| A_{d}|| \leq c_{d} k_{0} \ldots k_{d-1}\left(\varepsilon_{0}+\ldots+\varepsilon_{d-1}\right) \max \left|A_{i}\right|,
$$

for some constant $c_{d}$ which depends only on $d$.

Proof of Proposition 3.1. We denote $m=\max \left|A_{i}\right|$ and assume by induction that the proposition holds for $j-1$ (and any $\ell$ ), i.e. that

$$
\left|F^{j}\left(A_{0}, \ldots, A_{\ell}\right)-\frac{k_{0} k_{1} \ldots k_{j-2} k_{j-1}^{\ell-j+1}}{n^{\ell}} \prod_{i=0}^{\ell}\right| A_{i}|| \leq c_{j-1, \ell} m k_{0} k_{1} \ldots k_{j-2} k_{j-1}^{\ell-j+1}\left(\varepsilon_{0}+\ldots+\varepsilon_{j-1}\right) .
$$

For $j=0$ this indeed holds, in the sense that

$$
\left|F^{0}\left(A_{0}, \ldots, A_{\ell}\right)-\frac{k_{-1}^{\ell}}{n^{\ell}} \prod_{i=0}^{\ell}\right| A_{i}||=0 .
$$

Let us denote by $\mathcal{E}$ the discrepancy ||$F^{j+1}\left(A_{0}, \ldots, A_{\ell}\right)\left|-\frac{k_{0} k_{1} \ldots k_{j-1} k_{j}^{\ell-j}}{n^{\ell}} \prod_{i=0}^{\ell}\right| A_{i}||$. Combining the Descent Lemma with (3.5) (or (3.6), for $j=0$ ) multiplied by $\left(\frac{k_{j}}{k_{j-1}}\right)^{\ell-j}$ gives

$$
\begin{aligned}
\mathcal{E} \leq & (\ell-j) k_{j}^{\ell-j}\left(\varepsilon_{j}+\varepsilon_{j-1}\right) \sqrt{\left|F\left(A_{0}, \ldots, A_{j}\right)\right|\left|F\left(A_{\ell-j}, \ldots, A_{\ell}\right)\right|} \\
& +c_{j-1, \ell} m k_{0} k_{1} \ldots k_{j-1} k_{j}^{\ell-j}\left(\varepsilon_{0}+\ldots+\varepsilon_{j-1}\right) .
\end{aligned}
$$


To bound $\left|F\left(A_{0}, \ldots, A_{j}\right)\right|=\left|F^{j}\left(A_{0}, \ldots, A_{j}\right)\right|$ we use (3.5) with $\ell=j$, which gives

$$
\begin{aligned}
\left|F^{j}\left(A_{0}, \ldots, A_{j}\right)\right| & \leq \frac{k_{0} \ldots k_{j-1}}{n^{j}} \prod_{i=0}^{j}\left|A_{i}\right|+c_{j-1, j} m k_{0} \ldots k_{j-1}\left(\varepsilon_{0}+\ldots+\varepsilon_{j-1}\right) \\
& \leq\left[1+c_{j-1, j}\left(\varepsilon_{0}+\ldots+\varepsilon_{j-1}\right)\right] m k_{0} \ldots k_{j-1} \\
& \leq\left(1+j c_{j-1, j}\right) m k_{0} \ldots k_{j-1} .
\end{aligned}
$$

(here we used $\varepsilon_{i}<1$, but any bound on the $\varepsilon_{i}$ would do). The same holds for $\left|F\left(A_{\ell-j}, \ldots, A_{\ell}\right)\right|$, hence

$$
\begin{aligned}
\mathcal{E} \leq & (\ell-j) k_{j}^{\ell-j}\left(\varepsilon_{j}+\varepsilon_{j-1}\right)\left(1+j c_{j-1, j}\right) m k_{0} \ldots k_{j-1} \\
& +c_{j-1, \ell} m k_{0} k_{1} \ldots k_{j-1} k_{j}^{\ell-j}\left(\varepsilon_{0}+\ldots+\varepsilon_{j-1}\right) \\
= & m k_{0} k_{1} \ldots k_{j-1} k_{j}^{\ell-j}\left[c_{j-1, \ell}\left(\varepsilon_{0}+\ldots+\varepsilon_{j-1}\right)+(\ell-j)\left(1+j c_{j-1, j}\right)\left(\varepsilon_{j}+\varepsilon_{j-1}\right)\right] \\
\leq & \underbrace{\left[c_{j-1, \ell}+(\ell-j)\left(1+j c_{j-1, j}\right)\right]}_{c_{j, \ell}} m k_{0} k_{1} \ldots k_{j-1} k_{j}^{\ell-j}\left(\varepsilon_{0}+\ldots+\varepsilon_{j}\right) .
\end{aligned}
$$

as desired.

\section{Applications}

The following notion of geometric expansion for graphs and complexes originates in Gromov's work [Gro10] (see also [FGL $\left.{ }^{+} 12, \mathrm{MW} 11\right]$ ):

Definition 4.1. Let $X$ be a $d$-dimensional simplicial complex. The geometric overlap of $X$ is

$$
\text { overlap } X=\min _{\varphi: V \rightarrow \mathbb{R}^{d}} \max _{x \in \mathbb{R}^{d}} \frac{\#\left\{\sigma \in X^{d} \mid x \in \operatorname{conv}\{\varphi(v) \mid v \in \sigma\}\right\}}{\left|X^{d}\right|} .
$$

In other words, $X$ has overlap $\geq \varepsilon$ if for every simplicial mapping of $X$ into $\mathbb{R}^{d}$ (a mapping induced linearly by the images of the vertices), some point in $\mathbb{R}^{d}$ is covered by at least an $\varepsilon$-fraction of the $d$-cells of $X$.

A theorem of Pach [Pac98] relates combinatorial expansion and geometric overlap, and allows us to prove the following:

Proposition 4.2. If $X$ is a d-dimensional $(\bar{k}, \bar{\varepsilon})$-expander then

$$
\text { overlap } X>\frac{\mathcal{P}_{d} d !}{2^{d}}\left[\left(\frac{\mathcal{P}_{d}}{d+1}\right)^{d}-c_{d}\left(\varepsilon_{0}+\ldots+\varepsilon_{d-1}\right)\right],
$$

where $\mathcal{P}_{d}$ is Pach's constant [Pac98], and $c_{d}$ is the constant from Theorem 1.1 (both depend only on $d)$.

In particular, a family of $d$-complexes which have $\varepsilon_{0}+\ldots+\varepsilon_{d-1}$ small enough is a family of geometric expanders. For the proof of Proposition 4.2 we shall need the following lemma, which relates the Laplace spectrum to cell density: 
Lemma 4.3. Let $X$ be a d-complex with $\beta_{j}=0$ for $j<d$, and let $\lambda_{j}$ be the average nontrivial eigenvalue of $\Delta_{j}^{+}$, for $-1 \leq j<d$ (in particular $\lambda_{-1}=n$ ). For any $0 \leq m<d$ the average degree of an $m$-cell is

$$
\operatorname{avg}\left\{\operatorname{deg} \sigma \mid \sigma \in X^{m}\right\}=\lambda_{m}\left(1-\frac{m+1}{\lambda_{m-1}}\right),
$$

and the number of $m$-cells is

$$
\left|X^{m}\right|=\frac{\lambda_{m-1}}{m+1} \cdot \prod_{j=-1}^{m-2}\left(\frac{\lambda_{j}}{j+2}-1\right)=\frac{\lambda_{m-1}(n-1)}{m+1} \cdot \prod_{j=0}^{m-2}\left(\frac{\lambda_{j}}{j+2}-1\right) .
$$

Proof. Since the trivial spectrum of $\Delta_{j}^{+}$consists of zeros,

$$
\left|X^{m}\right|=\frac{1}{m+1} \sum_{\sigma \in X^{m-1}} \operatorname{deg} \sigma=\frac{1}{m+1} \operatorname{tr} D_{m-1}=\frac{1}{m+1} \operatorname{tr} \Delta_{m-1}^{+}=\frac{\lambda_{m-1}}{m+1} \operatorname{dim} Z_{m-1} .
$$

Thus, (4.2) is equivalent to the assertion that

$$
\operatorname{dim} Z_{m-1}=\prod_{j=-1}^{m-2}\left(\frac{\lambda_{j}}{j+2}-1\right)
$$

This is true for $m=0$, and by induction, together with the triviality of the $(m-2)$-th homology we find that

$$
\begin{gathered}
\operatorname{dim} Z_{m-1}=\operatorname{dim} \Omega^{m-1}-\operatorname{dim} B_{m-2}=\left|X^{m-1}\right|-\operatorname{dim} Z_{m-2} \\
=\frac{\lambda_{m-2}}{m} \prod_{j=-1}^{m-3}\left(\frac{\lambda_{j}}{j+2}-1\right)-\prod_{j=-1}^{m-3}\left(\frac{\lambda_{j}}{j+2}-1\right)=\prod_{j=-1}^{m-2}\left(\frac{\lambda_{j}}{j+2}-1\right)
\end{gathered}
$$

as desired. Formula (4.1) follows from (4.2), as avg $\left\{\operatorname{deg} \sigma \mid \sigma \in X^{m}\right\}=(m+2)\left|X^{m+1}\right| /\left|X^{m}\right|$.

We can now proceed:

Proof of Proposition 4.2. Let $\varphi$ be a simplicial map $X \rightarrow \mathbb{R}^{d}$, and divide $V=X^{0}$ arbitrarily into parts $P_{0}, \ldots, P_{d+1}$ of equal size $\left|P_{i}\right|=\frac{n}{d+1}$. Pach's theorem then states that there exist $Q_{i} \subseteq P_{i}$ of size $\left|Q_{i}\right|=\mathcal{P}_{d}\left|P_{i}\right|$ and a point $x \in \mathbb{R}^{d+1}$, such that $x \in \operatorname{conv}\{\varphi(v) \mid v \in \sigma\}$ for all $\sigma \in F\left(Q_{0}, \ldots, Q_{d}\right)$. Denoting $\mathcal{K}=k_{0} \cdot \ldots \cdot k_{d-1}$ and $\mathcal{E}=\varepsilon_{0}+\ldots+\varepsilon_{d-1}$, we have by Theorem 1.1

$$
\left|F\left(Q_{0}, \ldots, Q_{d}\right)\right| \geq \frac{\mathcal{K}}{n^{d}}\left(\frac{\mathcal{P}_{d} n}{d+1}\right)^{d+1}-\frac{c_{d} \mathcal{P}_{d} n \mathcal{K} \mathcal{E}}{d+1}=\frac{\mathcal{K} \mathcal{P}_{d} n}{d+1}\left[\left(\frac{\mathcal{P}_{d}}{d+1}\right)^{d}-c_{d} \mathcal{E}\right],
$$

and by the lemma above

$$
\left|X^{d}\right|=\frac{\lambda_{d-1}}{d+1} \cdot \prod_{j=-1}^{d-2}\left(\frac{\lambda_{j}}{j+2}-1\right) \leq \prod_{j=-1}^{d-1} \frac{\lambda_{j}}{j+2} \leq n \prod_{j=0}^{d-1} \frac{k_{j}\left(1+\varepsilon_{j}\right)}{j+2}<\frac{2^{d} n \mathcal{K}}{(d+1) !} .
$$

This means $x$ is covered by at least a $\frac{\mathcal{P}_{d} d !}{2^{d}}\left(\left(\frac{\mathcal{P}_{d}}{d+1}\right)^{d}-c_{d} \mathcal{E}\right)$-fraction of the $d$-cells, and as this is true for all $\varphi$ the proposition follows. 
We turn our attention to colorings. We say that a $d$-complex $X$ is $c$-colorable if there is a coloring of its vertices by $c$ colors so that no $d$-cell is monochromatic. The chromatic number of $X$, denoted $\chi(X)$, is the smallest $c$ for which $X$ is $c$-colorable. We will use the mixing property to show that spectral expansion implies a chromatic bound, as is done for graphs in [LPS88]. These results are weaker than Hoffman's chromatic bound for graphs [Hof70], as they require a two-sided spectral bound, and the chromatic bound obtained is not optimal. A chromatic bound for complexes which does generalize Hoffman's result was recently obtained in [Gol13].

Proposition 4.4. If $X$ is a d-dimensional $(\bar{k}, \bar{\varepsilon})$-expander, then

$$
\chi(X) \geq \frac{1}{(d+1) \sqrt[d]{c_{d}\left(\varepsilon_{0}+\ldots+\varepsilon_{d-1}\right)}}
$$

where $c_{d}$ is the constant from Theorem 1.1.

Proof. Coloring $X$ by $\chi=\chi(X)$ colors, there is necessarily a monochromatic set of vertices of size at least $\frac{n}{\chi}$. Take $\frac{n}{\chi}$ of these vertices and partition them arbitrarily to $d+1$ sets $A_{0}, \ldots, A_{d}$ of equal size. As in a coloring there are no monochromatic $d$-cells we have $F\left(A_{0}, \ldots, A_{d}\right)=\varnothing$, so that Theorem 1.1 reads

$$
\frac{k_{0} \ldots k_{d-1}}{n^{d}} \prod_{i=0}^{d}\left|A_{i}\right| \leq c_{d} k_{0} \ldots k_{d-1}\left(\varepsilon_{0}+\ldots+\varepsilon_{d-1}\right) \max \left|A_{i}\right|,
$$

and since $\left|A_{i}\right|=\frac{n}{\chi \cdot(d+1)}$, the conclusion follows.

\subsection{Ideal expanders}

Let us say that $X$ is an ideal $\bar{k}$-expander if it is a $\left(j, k_{j}, 0\right)$-expander for $0 \leq j<d$. In this case, the Descent Lemma tell us that

$$
F^{j+1}\left(A_{0}, \ldots, A_{\ell}\right)=\left(\frac{k_{j}}{k_{j-1}}\right)^{\ell-j}\left|F^{j}\left(A_{0}, \ldots, A_{\ell}\right)\right|,
$$

and the number of $j$-galleries between disjoint sets of vertices is completely determined by their sizes:

$$
\left|F^{j}\left(A_{0}, \ldots, A_{\ell}\right)\right|=\frac{k_{0} k_{1} \ldots k_{j-2} k_{j-1}^{\ell-j+1}}{n^{\ell}} \prod_{i=0}^{\ell}\left|A_{i}\right|
$$

(in particular, $\left.\left|F\left(A_{0}, \ldots, A_{d}\right)\right|=\frac{k_{0} \ldots k_{d-1}}{n^{d}}\left|A_{0}\right| \ldots\left|A_{d}\right|\right)$. For $k_{j}=\left\{\begin{array}{cc}n & 0 \leq j<m \\ 0 & m \leq j<d\end{array}\right.$, an example of an ideal $\bar{k}$-expander is given by $K_{n}^{(m)}$, the $m$-th skeleton of the complete complex on $n$ vertices. For this complex (4.3) holds trivially, and perhaps disappointingly, these are the only examples of ideal expanders: if $X$ is an ideal $\bar{k}$-expander on $n$ vertices, and $X^{(j)}=K_{n}^{(j)}$ (which holds for $j=0$ ), one has $k_{0}=\ldots=k_{j-1}=n$, and also $k_{j} \leq n$ by [PRT13, prop. 3.2(2)]. For vertices $v_{0}, \ldots, v_{j+1},\left|F\left(\left\{v_{0}\right\}, \ldots,\left\{v_{j+1}\right\}\right)\right|=\frac{k_{0} \ldots k_{j}}{n^{j+1}} \in\{0,1\}$ then forces either $k_{j}=n$, which implies that $X^{(j+1)}=K_{n}^{(j+1)}$ as well, or $k_{j}=0$, which means that $X$ has no $(j+1)$-cells at all.

While ideal $\bar{k}$-expanders do not actually exist, save for the trivial examples $\bar{k}=(n, \ldots, n, 0, \ldots)$, they provide a conceptual way to think of expanders in general: $(\bar{k}, \bar{\varepsilon})$-expanders spectrally approximate the ideal (nonexistent) $\bar{k}$-expander, and the mixing lemma asserts that they also combinatorially approximate it. This point of view seems close in spirit to that of spectral sparsification [ST11], which proved to be fruitful in both graphs and complexity theory. 


\section{Questions}

Several natural questions arise from this study:

- In [GW12] it is shown that random complexes in the Linial-Meshulam model [LM06] have spectral concentration for appropriate parameters (see also [PRT13, §4.5]). These are complexes with a complete skeleton, which are high-dimensional analogues of Erdôs-Rényi graphs. Is there a similar model for general complexes, for which the skeletons are not complete (preferably, where the expected degrees of cells are only logarithmic in the number of vertices), with concentrated spectrum?

- A well known source of excellent expanders are random regular graphs (see, e.g. [Pud12, Fri08]). Can one construct a model for random regular complexes, and are these complexes high-dimensional expanders? This is interesting even for a weak notion of regularity, such as having a bounded fluctuation of degrees, or having all links of vertices isomorphic.

- Ramanujan graphs, constructed in [LPS88, Mar88, MSS13] are another source of optimal expanders. Ramanujan complexes, their higher dimensional counterparts, were defined and studied in [CSŻ03, Li04, LSV05], but as yet not from the point of view of the Hodge Laplacian. It is natural to conjecture that they form spectral, and thus combinatorial expanders, as in the case of graphs.

- In [PRT13] a generalization for the discrete Cheeger inequality is given, for complexes with a complete skeleton. Can this result be generalized to arbitrary complexes?

- Can one prove a converse to the Expander Mixing Lemma in general dimension, as is done for graphs in [BL06]?

\section{References}

[ABM05] R. Aharoni, E. Berger, and R. Meshulam, Eigenvalues and homology of flag complexes and vector representations of graphs, Geometric and functional analysis 15 (2005), no. 3, 555566.

[AC88] N. Alon and F.R.K. Chung, Explicit construction of linear sized tolerant networks, Discrete Mathematics 72 (1988), no. 1-3, 15-19.

[Alo86] N. Alon, Eigenvalues and expanders, Combinatorica 6 (1986), no. 2, 83-96.

[AM85] N. Alon and V.D. Milman, $\lambda_{1}$, isoperimetric inequalities for graphs, and superconcentrators, Journal of Combinatorial Theory, Series B 38 (1985), no. 1, 73-88.

[BL06] Y. Bilu and N. Linial, Lifts, discrepancy and nearly optimal spectral gap, Combinatorica 26 (2006), no. 5, 495-519.

[BMS93] R. Beigel, G. Margulis, and D.A. Spielman, Fault diagnosis in a small constant number of parallel testing rounds, Proceedings of the fifth annual ACM symposium on Parallel algorithms and architectures, ACM, 1993, pp. 21-29.

[CSŻ03] D.I. Cartwright, P. Solé, and A. Żuk, Ramanujan geometries of type $\tilde{A}_{n}$, Discrete mathematics 269 (2003), no. 1, 35-43.

[DKM09] A. Duval, C. Klivans, and J. Martin, Simplicial matrix-tree theorems, Transactions of the American Mathematical Society 361 (2009), no. 11, 6073-6114.

[Dod84] J. Dodziuk, Difference equations, isoperimetric inequality and transience of certain random walks, Trans. Amer. Math. Soc 284 (1984).

[Eck44] B. Eckmann, Harmonische funktionen und randwertaufgaben in einem komplex, Commentarii Mathematici Helvetici 17 (1944), no. 1, 240-255.

$\left[\mathrm{FGL}^{+} 12\right]$ J. Fox, M. Gromov, V. Lafforgue, A. Naor, and J. Pach, Overlap properties of geometric expanders, J. Reine Angew. Math. 671 (2012), 49-83.

[FP87] J. Friedman and N. Pippenger, Expanding graphs contain all small trees, Combinatorica 7 (1987), no. 1, 71-76. 
[Fri98] J. Friedman, Computing Betti numbers via combinatorial laplacians, Algorithmica 21 (1998), no. 4, 331-346.

[Fri08] A proof of Alon's second eigenvalue conjecture and related problems, Mem. Amer. Math. Soc. 195 (2008), no. 910, viii+100. MR 2437174 (2010e:05181)

[Gar73] H. Garland, p-adic curvature and the cohomology of discrete subgroups of p-adic groups, The Annals of Mathematics 97 (1973), no. 3, 375-423.

[Gol13] K. Golubev, On the chromatic number of a simplicial complex, arXiv preprint arXiv:1306.4818 (2013).

[Gro10] M. Gromov, Singularities, expanders and topology of maps. part 2: From combinatorics to topology via algebraic isoperimetry, Geometric And Functional Analysis 20 (2010), no. 2, 416-526.

[GW12] A. Gundert and U. Wagner, On laplacians of random complexes, Proceedings of the 2012 symposuim on Computational Geometry, ACM, 2012, pp. 151-160.

[Hof70] A.J. Hoffman, On eigenvalues and colorings of graphs, Graph theory and its applications (1970), 79-91.

[KRS00] W. Kook, V. Reiner, and D. Stanton, Combinatorial Laplacians of matroid complexes, Journal of the American Mathematical Society 13 (2000), no. 1, 129-148.

[Li04] W.C.W. Li, Ramanujan hypergraphs, Geometric and Functional Analysis 14 (2004), no. 2, 380-399.

[LM06] N. Linial and R. Meshulam, Homological connectivity of random 2-complexes, Combinatorica 26 (2006), no. 4, 475-487.

[LPS88] A. Lubotzky, R. Phillips, and P. Sarnak, Ramanujan graphs, Combinatorica 8 (1988), no. 3, 261-277.

[LSV05] A. Lubotzky, B. Samuels, and U. Vishne, Ramanujan complexes of type $\tilde{A}_{d}$, Israel Journal of Mathematics 149 (2005), no. 1, 267-299.

[Mar88] G.A. Margulis, Explicit group-theoretical constructions of combinatorial schemes and their application to the design of expanders and concentrators, Problemy Peredachi Informatsii 24 (1988), no. 1, 51-60.

[MSS13] A. Marcus, D.A. Spielman, and N. Srivastava, Interlacing families I: Bipartite Ramanujan graphs of all degrees, arXiv preprint arXiv:1304.4132 (2013).

[MW11] J. Matoušek and U. Wagner, On Gromov's method of selecting heavily covered points, Arxiv preprint arXiv:1102.3515 (2011).

[Pac98] J. Pach, A Tverberg-type result on multicolored simplices, Computational Geometry 10 (1998), no. 2, 71-76.

[PR12] O. Parzanchevski and R. Rosenthal, Simplicial complexes: spectrum, homology and random walks, arXiv preprint arXiv:1211.6775 (2012).

[PRT13] O. Parzanchevski, R. Rosenthal, and R.J. Tessler, Isoperimetric inequalities in simplicial complexes, Combinatorica, To appear (2013), arXiv:1207.0638.

[Pud12] D. Puder, Expansion of random graphs: New proofs, new results, arXiv preprint arXiv:1212.5216 (2012).

[ST11] D.A. Spielman and S.H. Teng, Spectral sparsification of graphs, SIAM Journal on Computing 40 (2011), no. 4, 981-1025.

[Tan84] R.M. Tanner, Explicit concentrators from generalized n-gons, SIAM Journal on Algebraic and Discrete Methods 5 (1984), 287.

[Tao11] T. Tao, Basic theory of expander graphs, http://terrytao.wordpress.com/2011/12/02/ 245b-notes-1-basic-theory-of-expander-graphs/, 2011.

[Żuk96] A. Żuk, La propriété (T) de Kazhdan pour les groupes agissant sur les polyedres, Comptes rendus de l'Académie des sciences. Série 1, Mathématique 323 (1996), no. 5, 453-458.

SChool of Mathematics

InSTITUTE FOR ADVANCEd StUdy

PRINCETON, NJ 08540

E-mail: parzan@ias.edu 